\title{
Nachwuchs anfällig für Drogen?
}

\section{Hier steht eine Anzeige.}

Kinder von Müttern, die in der Schwangerschaft wenig oder kein Fleisch gegessen haben, greifen als Jugendliche häufiger zu Alkohol, Zigaretten und Cannabis.

Der Zusammenhang zwischen vegetarischer Ernährung in der Schwangerschaft und Suchtmittelkonsum beim Nachwuchs wurde in einer prospektiven Studie bei 5109 Jugendlichen untersucht. Jeweils etwa 10\% der 15-Jährigen gaben an, Probleme mit Alkohol zu haben, jede Woche Zigaretten zu rauchen oder ab und an Cannabis zu konsumieren. Diese drei Formen von Substanzmissbrauch fanden sich umso häufiger, je weniger Fleisch die Mutter während der Schwangerschaft gegessen hatte. Wenn pro 14 Tage maximal vier Portionen Fleisch verzehrt wurden, war verglichen mit mindestens einer Fleischmahlzeit pro Tag das Risiko für Alkohol-, Zigaretten- und Cannabiskonsum etwa verdoppelt (Odds Ratios: 1,7, 1,9 und 2,7).

Hibbeln J et al. Alcohol Clin Exp Res 2017; doi:10.1111/acer.13494

Megastudie aus den USA

\section{Bariatrische Op. senkt Krebsrisiko}

Die bariatrische Chirurgie ist bei Schwergewichtigen offenbar in der Lage, das Risiko für Krebs deutlich zu senken. In einer US-Kohortenstudie profitierten vor allem adipöse Frauen von dem Eingriff.

An der retrospektiven Kohortenstudie des Forscherteams um Dr. Daniel P. Schauer von der Universität Cincinnati haben insgesamt 88.679 adipöse Patienten teilgenommen. 22.198 Teilnehmer erhielten eine bariatrische Operation, und zwar überwiegend als Magenbypass- oder Schlauchmagen-Op. Diesen gegenübergestellt wurden 66.481 nicht operierte Kontrollpatienten. Was sich nach einer mittleren Beobachtungszeit von dreieinhalb Jahren zeigte, war ein statistisch signifikanter Vorteil bei den operierten Patienten: Bei ihnen war das Risiko, irgendeine Krebsform zu entwickeln, um rund 33\% niedriger als bei den Nicht-Operierten. Wer von dem Eingriff vor allem profitiert hatte, waren die Frauen. Nach der Op. war bei ihnen das Krebsrisiko gegenüber der (weiblichen) Kontrollgruppe um 36\% gesunken. Bei Männern war der Unterschied dagegen nicht signifikant. 